\title{
Effect of projectile on incomplete fusion reactions at low energies
}

\author{
Vijay R. Sharma ${ }^{1, \star}$, Mohd. Shuaib², Abhishek Yadav ${ }^{1}$, Pushpendra P. Singh ${ }^{3}$, Manoj K. Sharma ${ }^{4}$, R. Kumar ${ }^{1}$, \\ Devendra P. Singh ${ }^{5}$, B. P. Singh ${ }^{2, \star \star}$, S. Muralithar ${ }^{1}$, R. P. Singh ${ }^{1}$, R. K. Bhowmik ${ }^{1}$, and R. Prasad ${ }^{2}$ \\ ${ }^{1}$ NP-Group, Inter University Accelerator Centre, New Delhi - 110 067, India \\ ${ }^{2}$ Department of Physics, Aligarh Muslim University, Aligarh 202 002, India \\ ${ }^{3}$ Department of Physics, Indian Institute of Technology Ropar, Punjab 140 001, India \\ ${ }^{4}$ Physics Department, S. V. College, Aligarh - 202 001, India \\ ${ }^{5}$ Department of Physics, University of Petroleum and Energy Studies, Dehradun - 248007 , India
}

\begin{abstract}
Present work deals with the experimental studies of incomplete fusion reaction dynamics at energies as low as $\approx 4-7 \mathrm{MeV} / \mathrm{A}$. Excitation functions populated via complete fusion and/or incomplete fusion processes in ${ }^{12} \mathrm{C}+{ }^{175} \mathrm{Lu}$, and ${ }^{13} \mathrm{C}+{ }^{169} \mathrm{Tm}$ systems have been measured within the framework of PACE4 code. Data of excitation function measurements on comparison with different projectile-target combinations suggest the existence of ICF even at slightly above barrier energies where complete fusion (CF) is supposed to be the sole contributor, and further demonstrates strong projectile structure dependence of ICF. The incomplete fusion strength functions for ${ }^{12} \mathrm{C}+{ }^{175} \mathrm{Lu}$, and ${ }^{13} \mathrm{C}+{ }^{169} \mathrm{Tm}$ systems are analyzed as a function of various physical parameters at a constant $\mathrm{v}_{\text {rel }} \approx 0.053 \mathrm{c}$. It has been found that one neutron $(1 \mathrm{n})$ excess projectile ${ }^{13} \mathrm{C}$ (as compared to ${ }^{12} \mathrm{C}$ ) results in less incomplete fusion contribution due to its relatively large negative $\alpha$-Q-value, hence, $\alpha$ Q-value seems to be a reliable parameter to understand the ICF dynamics at low energies. In order to explore the reaction modes on the basis of their entry state spin population, the spin distribution of residues populated via $\mathrm{CF}$ and/or ICF in ${ }^{16} \mathrm{O}+{ }^{159} \mathrm{~Tb}$ system has been done using particle- $\gamma$ coincidence technique. CF- $\alpha$ and ICF- $\alpha$ channels have been identified from backward (B) and forward (F) $\alpha$-gated $\gamma$ spectra, respectively. Reaction dependent decay patterns have been observed in different $\alpha$ emitting channels. The CF channels are found to be fed over a broad spin range, however, ICF- $\alpha$ channels was observed only for high-spin states. Further, the existence of incomplete fusion at low bombarding energies indicates the possibility to populate high spin states.
\end{abstract}

\section{Introduction}

The study of breakup of heavy ions followed by the fusion of one of the fragments with the target has gained a resurgent interest. Several authors reported the competition of complete fusion (CF) and incomplete fusion (ICF) reactions at energies slightly above the Coulomb barrier [1-10], and a substantial ICF fraction has been observed at these energies. In general, the complete fusion $(\mathrm{CF})$ process is considered to be the sole contributor to the total fusion cross section at low energies i.e. starting from threshold to $7 \mathrm{MeV} / \mathrm{A}$.

Though, several studies and models [11-18] are developed to understand ICF phenomenon, but the dynamics of ICF at low incident energies is still not fairly understood and thus continues to be an active area of investigations. High quality data for ICF processes has been obtained and reported so far worldwide [1-8, 19, 20], and conclusively demonstrate the low energy ICF, but are limited only for a few projectile-target combinations. Therefore, for better understanding of ICF dynamics, several inclusive experiments at the Inter-University Accelerator

\footnotetext{
${ }^{\star}$ e-mail: phy.vijayraj@gmail.com

${ }^{\star}$ e-mail: bpsinghamu@gmail.com
}

Center (IUAC), New Delhi, India were performed by our group $[5,7,8,21,22]$. Based on experimental findings an attempt has been made to correlate data with suitable set of physical parameters viz., (a) projectile energy, (b) mass-asymmetry of interacting partners $(\mu)$, (c) $\alpha$-Q value, and (d) input $\ell$ values. In the present work, the percentage fraction of ICF were deduced from the analysis of experimental excitation functions of individual reaction residues for different projectile-target combinations. This suggests at the studied energy regime projectile structure plays an important role to understand ICF dynamics. To understand the role of $\ell$ values, a particle- $\gamma$ coincidence experiments were performed and spin distribution of individual reaction residues have been measured. The spin distribution of various evaporation residues reveals that the mean input angular momenta $(\langle\ell>)$ associated with the ICF observable are found to be higher than those involved with $\mathrm{CF}$. Further, the occurrence of ICF is due to the influence of centrifugal potential in peripheral interactions, where driving angular momentum limits do not allow CF to occur.

The present paper is organized as; the experimental details are presented in section 2. The observation from our recent experiments are given in section 3, where the influence of ICF on CF and its dependencies on various en- 


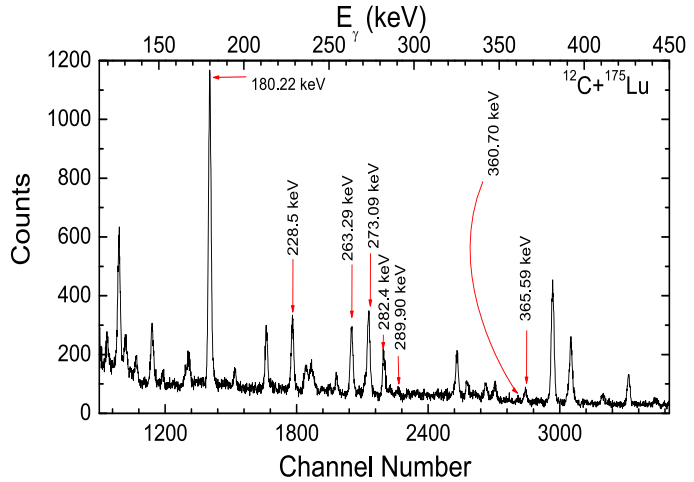

Figure 1. (Color online) Typical $\gamma$-ray spectrum of ${ }^{12} \mathrm{C}+{ }^{175} \mathrm{Lu}$ interaction at $\approx 78.36 \pm 1.08 \mathrm{MeV}$

trance channel parameters are demonstrated. Summary of the present work is given in Section 4.

\section{Experimental details}

To explore the dynamics of incomplete fusion reaction processes, experiments were performed using 15 UD pelletron accelerator of Inter University Accelerator Centre (IUAC), New Delhi, India. These experiments were performed using two different setups (a) General Purpose Scattering Chamber (GPSC) and (b) Gamma Detector Array (GDA) coupled with Charged Particle Detector Array (CPDA). The detailed methodology and experimental setups have been discussed in refs.[5, 7, 21, 22]. However, a short account of experimental conditions are given here for the ready reference.

In order to achieve information on the behavior of fraction of ICF observable on various entrance channel parameters, the value of incomplete fusion fraction $\left(\mathrm{F}_{I C F}\right)$ has been deduced as a function of various physical parameters (discussed in sec 3 ) in a separate set of experiment. In this experiment, absolute production cross-sections of individual reaction residues populated via $\mathrm{CF}$ and/or ICF in ${ }^{12} \mathrm{C}+{ }^{175} \mathrm{Lu}$, and ${ }^{13} \mathrm{C}+{ }^{169} \mathrm{Tm}$ systems have been measured at energies starting from threshold to $7 \mathrm{MeV} / \mathrm{A}[7,23]$. The target foils of isotopically pure $(99.9 \%){ }^{175} \mathrm{Lu}$, and ${ }^{169} \mathrm{Tm}$ along with in ${ }^{27} \mathrm{Al}$ catchers were prepared using rolling technique. The thickness of the samples were determined from the observed change in the energy of the $\alpha$ particles by using stopping power values and were found to $\approx 1.5$ to $\approx 2.5 \mathrm{mg} / \mathrm{cm}^{2}$ for both targets and $\approx 1.0$ to $\approx 3.0$ $\mathrm{mg} / \mathrm{cm}^{2}$ for Al-catchers. The thickness of the Al-catchers was chosen keeping in view the fact that even the most energetic residues produced due to the complete momentum transfer may be trapped in the catcher thickness. The irradiation has been carried out with a beam current $\approx 2-3$ pnA. The evaporation residues (ERs) produced during the irradiations were counted off-line with two pre-calibrated HPGe detectors. The ERs have been identified by their characteristic $\gamma$-lines and are further confirmed by decaycurve analysis. A part of $\gamma$-ray spectra obtained at $\mathrm{E}_{L a b}$ $=78.36 \pm 1.08 \mathrm{MeV}$ in ${ }^{12} \mathrm{C}+{ }^{175} \mathrm{Lu}$ system is shown in

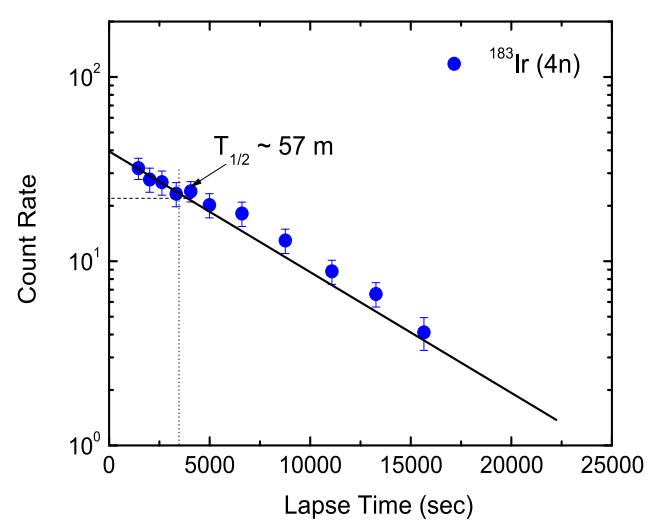

Figure 2. (Color online) Typical decay curve of ${ }^{183}$ Ir residue observed in ${ }^{12} \mathrm{C}+{ }^{175} \mathrm{Lu}$ interaction at $\approx 78.36 \pm 1.08 \mathrm{MeV}$ beam energy.

Fig.1. Some of the peaks corresponding to different reaction residues are marked. As a typical example, the observed decay curve for one of the Iridium isotopes ${ }^{183} \mathrm{Ir}$ $\left(\mathrm{T}_{1 / 2}=57 \mathrm{~min}\right)$ populated in ${ }^{12} \mathrm{C}+{ }^{175} \mathrm{Lu}$ system is shown in Fig. 2. Further, the production cross-sections $\left(\sigma_{R}\right)$ of ERs have been calculated using standard formulation [23]. The overall errors in the present measurement are estimated to be $\approx 10 \%$. A detailed discussion on error analysis is presented in Refs. [24].

To probe the role of $\ell$-values in the onset of ICF at low projectile energies, the spin-distributions (SDs) of $\mathrm{xn} / \mathrm{pxn} / \alpha \mathrm{xn}$ channels has been measured in ${ }^{16} \mathrm{O}+{ }^{159} \mathrm{~Tb}$ system using particle- $\gamma$ coincidence technique. The experiment was performed at four set of projectile energies viz. $\approx 83.5 \pm 1.5,88.5 \pm 1.5,93.5 \pm 1.5$ and $97.6 \pm 1.4 \mathrm{MeVs}$, using GDA alongwith CPDA facility of IUAC, New Delhi, India. The particle- $\gamma$ coincidences were recorded using 12 Compton suppressed, high resolution HPGe detectors installed at an angles $45^{\circ}, 99^{\circ}, 153^{\circ}$ with respect to the beam axis in Gamma Detector Array (GDA). It may be mentioned that there were four detectors at each of these angles in GDA set up. Further, the GDA set up is coupled with the Charge particle detector array (CPDA) which is a set of 14phoswich detectors housed in a $14 \mathrm{~cm}$ diameter scattering chamber. These particle detectors cover nearly $90 \%$ of the total solid angle. All 14 detectors of CPDA have been divided into the angular zones viz.; (i) Forward angle (F) $10^{0}$ to $60^{\circ}$, (ii) Sideways (S) $60^{\circ}$ to $120^{\circ}$, and (iii) Backward angle (B) $120^{\circ} 170^{\circ}$. Depending on the fast and slow components of the CPDA, proton and $\alpha$-particles in each angular ring can be identified. For the experiment, isotopically pure, self-supporting ${ }^{159} \mathrm{~Tb}(100 \%)$ target of thickness $\approx 1.5 \mathrm{mg} / \mathrm{cm}^{2}$ (prepared by rolling technique) has been bombarded with ${ }^{16} \mathrm{O}^{+7}$ beams delivered from 15UD Pelletron Accelerator. A prompt $\gamma$-ray spectra was recorded in event-by-event multi parameter mode, which includes different coincidences i.e. particles $(Z=1,2)$ detected in backward (B), forward (F) and $90^{\circ}(\mathrm{S})$-angles. Singles data have also been collected to identify xn-channels (pro- 
duced via $\mathrm{CF}$ ). Data analysis was done in two steps. In the first step, spin distributions of xn-channels have been measured by looking into singles spectra. For the identification of pxn-channels, backward (B)- $\alpha$-gated spectra has been subtracted from backward(B)-particles( $\mathrm{Z}=1,2)$ gated spectra to achieve proton-gated spectra. However, $\alpha \mathrm{xn}$-channels populated via $\mathrm{CF}$ have been identified from the backward (B)- $\alpha$-gated spectra. Further, the fast- $\alpha$ particles (which are expected to be emitted only in forward cone) produced via ICF have been identified from forward(F)- $\alpha$-gated spectra obtained by subtracting backward (B)- $\alpha$-gated spectra from forward(F)- $\alpha$-gated spectra. The intensity and area under the photo-peak (efficiency corrected) of the characteristic prompt $\alpha$-transitions were used to determine the relative production yield [21]

\section{Projectile Dependence of ICF: Recent Results}

In the present work, a systematic investigation have been done to understand the effect of various entrance channel parameters (i.e. projectile energy, entrance channel massasymmetry of interacting partners, $\alpha$-Q-value, and input $\ell$-values) on the onset and strength of incomplete fusion. Interpretation of the data and results are presented in this section.

\subsection{ICF dependence on incident energy}

The excitation functions (EFs), expected to be populated via $\mathrm{CF}$ and/or ICF mode have been measured for ${ }^{12} \mathrm{C}+{ }^{175} \mathrm{Lu}$, and ${ }^{13} \mathrm{C}+{ }^{169} \mathrm{Tm}$ systems at energies starting from the Coulomb barrier to $7 \mathrm{MeV} / \mathrm{A}$ and analyzed in the framework of statistical model code PACE4 to probe the energy dependence of ICF. Details of code PACE4 can be found in ref. [25]. In this code, the nuclear level density $(\mathrm{a}=\mathrm{A} / \mathrm{K})$ plays an important role which can be tuned to fit the experimental data. In order to choose the suitable value of level density to reproduce fusion EFs, different values of $K=8-12$ have been tested. The evaporation residues (ERs) populated via $\mathrm{xn} / \mathrm{pxnchannels} \mathrm{have} \mathrm{been}$ found to be in good agreement with that estimated from code PACE4. On the other hand, the experimental EFs of $\alpha$-emitting channels have been found to be significantly enhanced as compared to the PACE4 predictions. This enhanced value of $\alpha$ ERs may be attributed to ICF. To have the enhanced value of ERs (i.e. ICF contribution), experimental EFs of $\alpha$-emitting channels were subtracted from the PACE4 values $\left(\sigma_{I C F}=\sigma_{\exp }-\sigma_{P A C E 4}\right)$ [25]. For better insight into the onset and strength of ICF, the percentage fraction of ICF $\left(\mathrm{F}_{I C F}\right)$ has been deduced and is plotted as a function of projectile relative velocity $\left(\mathrm{v}_{\text {rel }}\right)$ [5] for ${ }^{12} \mathrm{C}+{ }^{175} \mathrm{Lu},{ }^{13} \mathrm{C}+{ }^{169} \mathrm{Tm}$ and ${ }^{12} \mathrm{C}+{ }^{169} \mathrm{Tm}$ systems in Fig.3. It may be mentioned that $\mathrm{F}_{I C F}$ defines empirical probability of ICF.

As shown in this figure, the $\mathrm{F}_{I C F}$ increases linearly with normalized projectile energies in terms of relative velocity of projectile (i.e., $\mathrm{v}_{\text {rel }}$ ). This suggests strong projectile energy dependence of ICF reactions. On the other

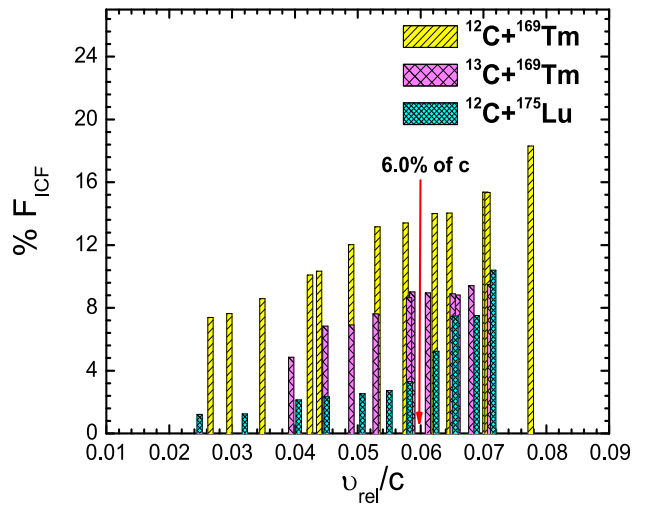

Figure 3. (Color online) The percentage fraction of ICF as a function of projectile relative velocity $\left(\mathrm{v}_{\text {rel }}\right)$ for ${ }^{12} \mathrm{C}+{ }^{175} \mathrm{Lu}$, ${ }^{12} \mathrm{C}+{ }^{169} \mathrm{Tm}$ and ${ }^{13} \mathrm{C}+{ }^{169} \mathrm{Tm}$ systems

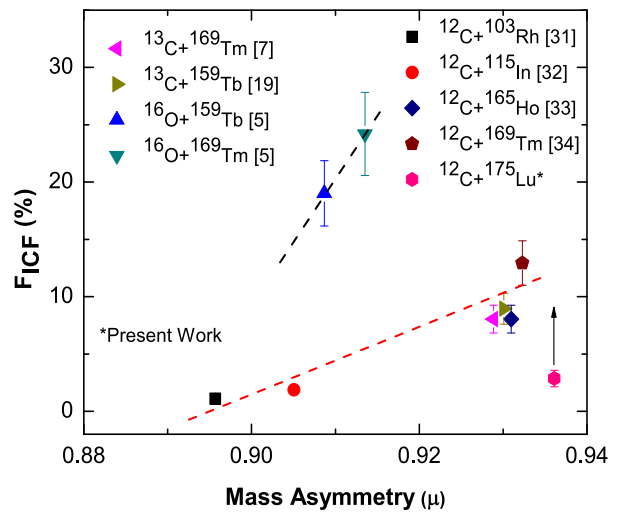

Figure 4. (Color online) The percentage fraction of ICF as a function of $\mu$ for 9 projectile-target combinations at a constant value of $\mathrm{v}_{\text {rel }} \approx 0.053 \mathrm{c}$. Dashed lines are drawn to guide the eyes.

hand, Morgenstern systematics [26, 27] suggests that ICF contributes significantly above $\mathrm{v}_{\text {rel }} \geq 0.06$ (6\% speed of light). However, in figure 3 , the values of $\mathrm{v}_{\text {rel }}$ are in the range from $\approx 2.7 \%$ of $\mathrm{c}$ to $\approx 7.8 \%$ of $\mathrm{c}$ for ${ }^{12} \mathrm{C}+{ }^{175} \mathrm{Lu}$, and ${ }^{12} \mathrm{C}+{ }^{169} \mathrm{Tm}$ systems and from $2.3 \%$ of $\mathrm{c}$ to $7.0 \%$ of $\mathrm{c}$ for ${ }^{13} \mathrm{C}+{ }^{169} \mathrm{Tm}$ system. Hence, the results presented in Fig.3 clearly demonstrate the onset of ICF at relatively lower value of $\mathrm{v}_{\text {rel }}$ i.e. $\mathrm{F}_{I C F}$ is significant at well below the proposed onset value of $\mathrm{v}_{r e l}$. Further, for the same target the value of $\mathrm{F}_{I C F}$ for ${ }^{12} \mathrm{C}$-projectile is lower than ${ }^{13} \mathrm{C}$ projectile for the entire measured energy range. The difference in $\mathrm{F}_{I C F}$ for two systems $\left({ }^{12} \mathrm{C},{ }^{13} \mathrm{C}+{ }^{169} \mathrm{Tm}\right)$ clearly points towards projectile structure and/or mass asymmetry effect on ICF.

\subsection{Projectile structure dependence of ICF}

To understand projectile structure and/or mass asymmetry $(\mu)$ dependence of ICF, the deduced percentage fraction of ICF for ${ }^{12} \mathrm{C}+{ }^{175} \mathrm{Lu}$, and ${ }^{13} \mathrm{C}+{ }^{169} \mathrm{Tm}$ systems alongwith 


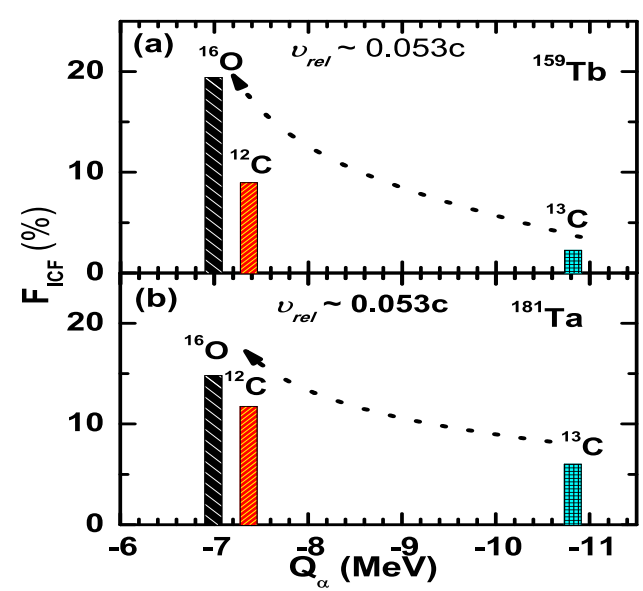

Figure 5. (Color online) Comparison of $\mathrm{F}_{I C F}$ on the basis of $\alpha$ $\mathrm{Q}$ value of the projectile at a constant $\mathrm{v}_{\text {rel }}=0.053 \mathrm{c}$ for different projectile-target combinations

7 projectile-target combinations are plotted as a function of mass asymmetry at a constant value of $\mathrm{v}_{\text {rel }} \approx 0.053$ in Fig.4. In order to understand projectile dependence on ICF more conclusively, data for the ${ }^{16} \mathrm{O}$ projectile is also added. It may be pointed out that ${ }^{12} \mathrm{C},{ }^{13} \mathrm{C}$ and ${ }^{16} \mathrm{O}$ are widely different projectiles and hence the probability of breakup depends on the input $\ell$-values imparted into system in peripheral interactions. As can be seen from Fig.4, the Morgensterns systematics does not explain the variation of $\mathrm{F}_{I C F}$ with $\mu$ for given systems. However, the value of $\mathrm{F}_{I C F}$ increases with $\mu$ for ${ }^{12} \mathrm{C},{ }^{13} \mathrm{C}$ projectiles and individually for ${ }^{16} \mathrm{O}$ projectile. It is interesting to note that the ${ }^{12} \mathrm{C},{ }^{13} \mathrm{C}+{ }^{169} \mathrm{Tm}$ systems is a more mass asymmetric $(\mu \approx$ $0.9337)$ system than ${ }^{16} \mathrm{O}+{ }^{169} \mathrm{Tm}$ system $(\mu \approx 0.9135)$, but the value of $\mathrm{F}_{I C F}$ is $\approx 18 \%$ higher than that observed for ${ }^{12} \mathrm{C},{ }^{13} \mathrm{C}+{ }^{169} \mathrm{Tm}$ system. The aforementioned observations based on 9 projectile-target combinations strongly contradict Morgensterns mass-asymmetry systematics, and suggest strong projectile dependence of ICF for alpha cluster projectiles. However projectile dependence of mass asymmetry is valid for same atomic number and different mass number projectiles (i.e. ${ }^{12} \mathrm{C},{ }^{13} \mathrm{C}$, etc. on the same target) is a question of interest. As can be seen in Fig 3 the values of $\mathrm{F}_{I C F}$ for the ${ }^{13} \mathrm{C}$ projectile are less than for the ${ }^{12} \mathrm{C}$ projectile in the studied energy range. The difference in $\mathrm{F}_{I C F}$ for two systems $\left({ }^{12} \mathrm{C},{ }^{13} \mathrm{C}+{ }^{169} \mathrm{Tm}\right)$ is clearly evident, and indicates the strong projectile dependence of $\mathrm{F}_{I C F}$ with some physical parameter other than $\mu$. In order to explore this issue, the ICF strength functions for ${ }^{12} \mathrm{C},{ }^{13} \mathrm{C}+{ }^{169} \mathrm{Tm}$ systems have been studied in terms of projectile $\alpha$-Q value.

\subsection{Projectile $\alpha-\mathbf{Q}$ value dependence of ICF}

The percentage fraction of ICF is analyzed in terms of projectile $\alpha \mathrm{Q}$-value for 6 projectile-target combinations. The value of $\mathrm{F}_{I C F}$ is plotted as a function of $Q_{\alpha}$ in Fig.5(a-b) at a constant $\mathrm{v}_{\text {rel }} \approx 0.053 \mathrm{c}$. As shown in this figure, the probability of ICF for the ${ }^{13} \mathrm{C}$ projectile is smaller than that for the ${ }^{12} \mathrm{C}$ projectile. This clearly point towards the

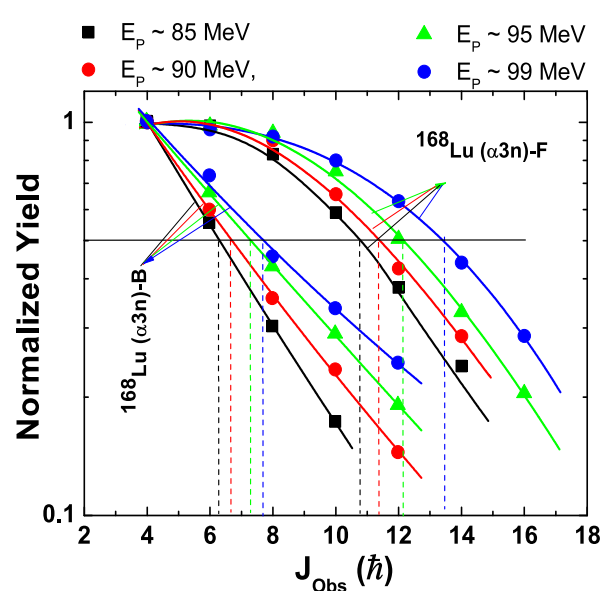

Figure 6. (Color online) Experimentally generated spindistributions of $\alpha 3 \mathrm{n}-(\mathrm{B})$, and $\alpha 3 \mathrm{n}-(\mathrm{F})$ channels. Lines and curves are the best fit to the experimental data points.

projectile structure effect. It may be pointed out that ${ }^{12} \mathrm{C}$ is a well known $\alpha$-cluster nucleus with $Q_{\alpha}=-7.37 \mathrm{MeV}$. However, ${ }^{13} \mathrm{C}$ has a larger $Q_{\alpha}(=-10.64 \mathrm{MeV})$ than ${ }^{12} \mathrm{C}$. The higher $\alpha$ Q-value for ${ }^{13} \mathrm{C}$ translates into the smaller breakup probability into constituent $\alpha$-clusters, resulting smaller ICF fraction than that found in ${ }^{12} \mathrm{C}$ induced reactions. In order to strengthen $\alpha \mathrm{Q}$-value dependence, the value of $\mathrm{F}_{I C F}$ for the ${ }^{16} \mathrm{O}\left(Q_{\alpha}=-7.16 \mathrm{MeV}\right)+{ }^{159} \mathrm{~Tb}$ system is found to be $18 \%$ which is reduced to only $3 \%$ for the ${ }^{13} \mathrm{C}\left(Q_{\alpha}=-10.64 \mathrm{MeV}\right)+{ }^{159} \mathrm{~Tb}$ system. Similar trend was observed for the ${ }^{181} \mathrm{Ta}$ target. Hence, from the data presented in this figure, it can be inferred that the $\alpha \mathrm{Q}$-value is an important entrance channel parameter which essentially dictates the probability of ICF.

\section{4 $\ell$ dependence of ICF}

In order to understand the physics of angular momentum involvement and de-excitation pattern of any residual nuclei, the spin distribution of various $\mathrm{CF}$ and ICF channels have been measured for ${ }^{16} \mathrm{O}+{ }^{159} \mathrm{~Tb}$ system at laboratory energies $\left(\mathrm{E}_{l a b}\right) \approx 83.5 \pm 1.5,88.5 \pm 1.5,93.5 \pm 1.5$ and $97.6 \pm 1.4 \mathrm{MeV}$ with beam intensities of $\approx 4-6 \mathrm{pnA}$. It is expected that in case of $\mathrm{CF}$ residues (high $\mathrm{E}^{*}$ and low $\ell$ ) the de-excitation pattern are more likely as statistical transitions, on the other hand, the ICF residues achieve low $\mathrm{E}^{*}$ (due to the involvement of partial degrees of excitations) and high $\ell$ (relatively higher values of impact parameters contribute to the high spin states) at a given projectile energy, therefore, the number of yrast - like transitions are much larger than that of statistical ones.

In view of the above, a comparison of the spin distribution pattern for the $\alpha 3 \mathrm{n}$ channel $\left({ }^{168} \mathrm{Lu}\right)$ populated via both $\mathrm{CF}$ mode $(\rightarrow$ identified in the backward (B) $\alpha$-gated spectra) and ICF mode ( $\rightarrow$ identified in the forward (F) $\alpha$-gated spectra) are plotted in Fig.6. As shown in this figure, there is a difference in the spin-distributions which indicate the involvement of entirely different mode of reactions in the production of these residues. The intensity of $\alpha 3 \mathrm{n}$ (B)- 


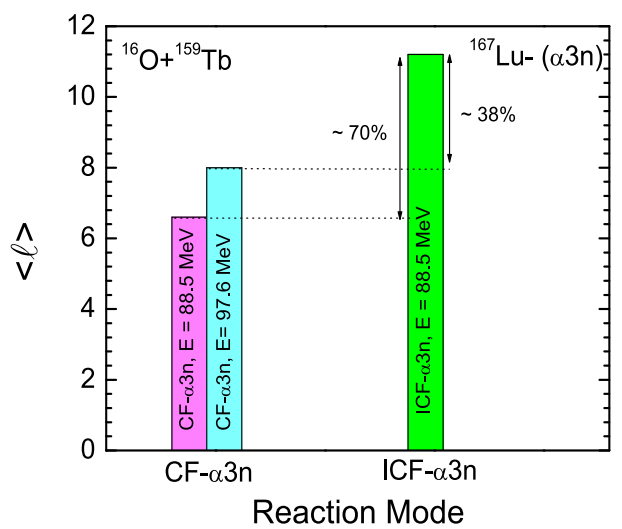

Figure 7. (Color online) The value of the mean angular momenta i.e., the value of $\langle\ell\rangle$ deduced from the best fitting procedure of spin distributions [21] involved in ${ }^{167} \mathrm{Lu}$ isotope populated via $\mathrm{CF}$ and ICF processes as a function of modes of reaction.

channels (CF residues) falls off rather quickly with observed spin $\left(\mathrm{J}_{o b s}\right)$, indicating strong feeding and/or broad spin population during the de-excitation of $\mathrm{CN}$. However, for $\alpha 3 \mathrm{n}(\mathrm{F})$-channels (ICF residues), the intensity appears to be almost constant up to a certain value of $\mathrm{J}_{o b s}$, and then decreases towards band head. This indicates the absence of feeding to the lowest members of the Yrast band and/or the population of low spin states are strongly hindered in ICF-channels.

Concerning the usefulness of incomplete fusion as a tool to populate high-spin states in final reaction products the mean input angular momenta $(<\ell>)$ associated with $\alpha 3 \mathrm{n}$ channel (populated via both CF and ICF modes) have been deduced as suggested in Ref [21] and is plotted in Fig.7. As can be seen in Fig. 7, the $\langle\ell>$ values involved in the production of ${ }^{168} \mathrm{Lu}$ via ICF- $\alpha 3 \mathrm{n}$ channel (i.e. $\approx$ $11.0 \hbar)$ is found to be $70 \%$ larger than that via CF- $\alpha 3 \mathrm{n}$ channel (i.e. $\approx 6.5 \hbar$ ) at the same projectile energy, i.e., $\mathrm{E}_{\text {lab }} \approx 88.5 \pm 1.5 \mathrm{MeV}$. On the other hand, the value of $\ell$ involved in ICF- $\alpha 3 \mathrm{n}$ channel (i.e. $\approx 10.5 \hbar$ ) at $83.5 \pm 1.5$ $\mathrm{MeV}$ is found to be $\approx 40 \%$ more than that involved in CF$\alpha 3 \mathrm{n}$ channel (i.e. $\approx 7.5 \hbar$ ) even at highest experimental incident laboratory energy $\left(\mathrm{E}_{l a b}\right) \approx 97.6 \pm 1.4 \mathrm{MeV}$. This clearly demonstrates the involvement of higher $\ell$-values in the production of incomplete channels which may lead to the population of higher spin-states in the final reaction products which may not be possible otherwise. The present spin distribution measurement for ${ }^{16} \mathrm{O}+{ }^{159} \mathrm{~Tb}$ system supports earlier findings by Dracoulis et al [28], Lane et al [29], and Mullins et al [30] on the populatation of high spin states in final reaction products at low bombarding energies. Hence, ICF can be used as a spectroscopic tool to study the high spin states even at low energies.

\section{Summary}

This paper gives the recent experimental results for the excitation function and particle- $\gamma$ coincidence experiments.
The EFs for several residues populated via $\mathrm{CF}$ and/or ICF in ${ }^{12} \mathrm{C}+{ }^{175} \mathrm{Lu}$ and ${ }^{13} \mathrm{C}+{ }^{169} \mathrm{Tm}$ systems have been measured at energies starting from threshold to $\approx 7 \mathrm{MeV} /$ nucleon, and analyzed in the framework of statistical model code PACE4. A systematic analysis of ICF dependence on various entrance channel parameters has been performed. It has been found that ICF strongly depends on incident energy, projectile and target type, $\alpha$ Q-values. Results and analysis presented on projectile structure effects suggest more ICF fraction for less negative $\alpha$ Q-value projectiles. Results presented are in good agreement with the existing data.

The spin-distribution(s) associated with ICF are found to be originated from the narrow spin population, localized near and/or above to the critical angular momentum $\left(\ell_{c r i t}\right)$ for $\mathrm{CF}$, where a given direct- $\alpha$-fragment is emitted to release excess driving angular momenta. This reveals a competition from successively opened ICF channels for each value of $\ell$ above the $\ell_{\text {crit }}$ for normal fusion (CF). On the basis of the present analysis of experimental data, the mean angular momenta associated with ICF are found to be $\approx 70 \%$ higher than that for $\mathrm{CF}$ at the same energy. Further, the mean input angular momenta associated with ICF residues at lowest energy is $40 \%$ higher than that for the $\mathrm{CF}$ residue at the highest studied energy, this helps to understand the population of high spin states via ICF route.

\section{Acknowledgements}

The authors thank to the Director, IUAC, New Delhi, for providing all the necessary facilities to carry out this work. One of the author (VRS) thanks to DST for providing travel support.

\section{References}

[1] P. R. S. Gomes, R. Linares, J. Lubian, C. C. Lopes, E. N. Cardozo, B. H. F. Pereira, and I. Padron, Phys. Rev. C 84, 014615 (2011).

[2] K. Surendra Babu, R. Tripathi, K. Sudarshan, B. D. Shrivastava, A. Goswami, and B. S. Tomar, J. Phys. G 29, 1011 (2003).

[3] K. Sudarsan Babu et al., Nucl. Phys. A 739, 229 (2004).

[4] D.J. Hinde, M. Dasgupta, Phys. Rev. C 81, 064611 (2010), and the references therein.

[5] P. P. Singh, B. P. Singh, M. K. Sharma, Unnati, D. P. Singh, and R. Prasad, Phys. Rev. C 77, 014607 (2008).

[6] M. Dasgupta, D. J. Hinde, R. D. Butt, R. M. Anjos, A. C. Berriman, N. Carlin, P.R. S.Gomes, C. R. Morton, J. O. Newton, A. Szanto de Toledo, and K. Hagino, Phys. Rev. Lett. 82, 1395 (1999).

[7] Vijay R Sharma et al., Phys. Rev. C 89, 024608 (2014).

[8] Mohd. Shuaib, et al, Phys. Rev. C 94, 014613 (2016).

[9] K. Sudarshan, et al, Phys. Rev. C 69, 027603 (2004).

[10] R. Tripathi, et al, Eur. Phys. J. A 42: 25 (2009).

[11] I. Tserruya et al., Phys. Rev. Lett. 60, 14 (1988). 
[12] E. Takada, T. Shimoda, N. Takahashi, T. Yamaya, K. Nagatani, T. Udagawa, and T. Tamura, Phys. Rev. C 23, 772 (1981).

[13] J.Wilczynski, K. Siwek-Wilczynska, J. Van Driel, S. Gonggrijp, D. C. J. M. Hageman, R. V. F. Janssens, J. Lukasiak, R. H. Siemssen, and S. Y. Van Der Werf, Nucl. Phys. A 373, 109 (1982).

[14] M. Blann, Phys. Lett. 27, 337 (1971).

[15] J. P. Bondrof et al., Nucl. Phys. A 333, 285 (1980).

[16] B. G. Harvey, Phys. Lett. B 130, 373 (1983).

[17] B. G. Harvey, Nucl. Phys. A 444, 498 (1985).

[18] A. Y. Abul-Magd, Z. Phys. A 298, 143 (1980).

[19] A. Yadav, V. R. Sharma, P. P. Singh, R. Kumar, D. P. Singh, Unnati, M. K. Sharma, B. P. Singh, and R. Prasad, Phys. Rev. C 86, 014603 (2012), and references therein.

[20] Manoj K Sharma Physical Rev. C 91, 024608 (2015).

[21] Vijay R. Sharma, et al., J. Phys. G, Nucl. Part. Phys. 42055113 (2015).

[22] Pushpendra P. Singh, et al., Phys. Lett. B 671, 20 (2009).

[23] Vijay R. Sharma, Ph.D. Thesis, A.M. University, Aligarh-Unpublished, (2015).
[24] B. P. Singh, M. G. V. Sankaracharyulu, M. A. Ansari, H. D. Bhardwaj, and R. Prasad, Phys. Rev. C 47, 2055 (1993).

[25] O. B. Tarasov and D. Bazin, Nucl. Instrum. Methods Phys. Res., Sect. B 204, 174 (2003).

[26] H. Morgenstern, W. Bohne, W. Galster, and K. Grabisch, Z. Phys. A. Atomic Nuclei 324, 443 (1986).

[27] H. Morgenstern, W. Bohne, W. Galster, K. Grabisch, and A. Kyanowski, Z. Phys. A 313, 39 (1983); H. M. Morgenstern, W. Bohne, K. Grabisch, D. G. Kovar, and H. Lehr, Phys. Lett. B 113, 463 (1982).

[28] G.D. Dracoulis, et al., J. Phys. G, Nucl. Part. Phys. 23, 1191 (1997).

[29] G.J. Lane, G.D. Dracoulis, A.P. Byrne, A.R. Poletti, T.R. McGoram, Phys. Rev. C 60, 067301 (1999).

[30] S.M. Mullins, et al., Phys. Lett. B 393, 279 (1997); S.M. Mullins, et al., Phys. Rev. C 61, 044315 (2000).

[31] Bindu Kumar, et al., Phys. Rev. C 59, 2923 (1999).

[32] S. Mukherjee, et al., Int. J. Modern Phys. E 15, 237 (2006).

[33] S. Gupta, et al., Phys. Rev. C 61, 064613 (2000).

[34] P. P. Singh, et al., Jour. of Phys: Conf. Series 590, 012031 (2015). 